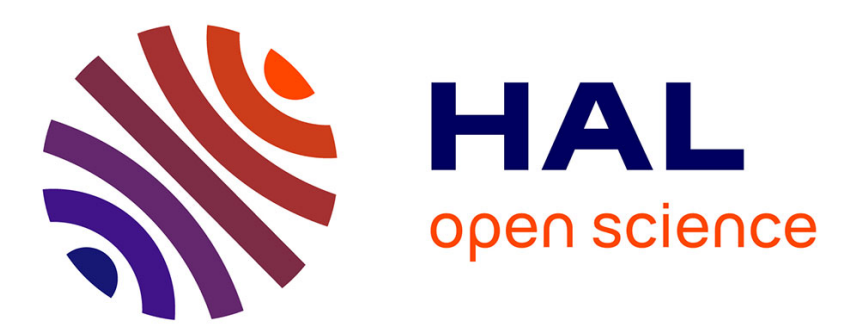

\title{
Comparison between numerical models for the simulation of moving contact lines
}

Dominique Legendre, Marco Maglio

\section{To cite this version:}

Dominique Legendre, Marco Maglio. Comparison between numerical models for the simulation of moving contact lines. Computers and Fluids, 2015, 113, pp.2-13. 10.1016/j.compfluid.2014.09.018 . hal-01340390

\author{
HAL Id: hal-01340390 \\ https://hal.science/hal-01340390
}

Submitted on 1 Jul 2016

HAL is a multi-disciplinary open access archive for the deposit and dissemination of scientific research documents, whether they are published or not. The documents may come from teaching and research institutions in France or abroad, or from public or private research centers.
L'archive ouverte pluridisciplinaire HAL, est destinée au dépôt et à la diffusion de documents scientifiques de niveau recherche, publiés ou non, émanant des établissements d'enseignement et de recherche français ou étrangers, des laboratoires publics ou privés. 


\section{OATAO}

\section{Open Archive TOULOUSE Archive Ouverte (OATAO)}

OATAO is an open access repository that collects the work of Toulouse researchers and makes it freely available over the web where possible.

This is an author-deposited version published in : http://oatao.univ-toulouse.fr/ Eprints ID : 15876

To link to this article : DOI:10.1016/j.compfluid.2014.09.018 URL : http://dx.doi.org/10.1016/j.compfluid.2014.09.018

To cite this version : Legendre, Dominique and Maglio, Marco Comparison between numerical models for the simulation of moving contact lines. (2015) Computers and Fluids, vol. 113. pp. 2 -13. ISSN 0045-7930

Any correspondence concerning this service should be sent to the repository administrator: staff-oatao@,listes-diff.inp-toulouse.fr 


\title{
Comparison between numerical models for the simulation of moving contact lines
}

\author{
D. Legendre*, M. Maglio \\ University of Toulouse, INPT-UPS, Institut de Mécanique des Fluides de Toulouse, France \\ CNRS, IMFT, Institut de Mécanique des Fluides de Toulouse, France
}

\begin{abstract}
A B S T R A C T
The aim of this study is to discus different numerically models for the simulation of moving contact lines in the context of a Volume of Fluid-Continuum Surface Force (VoF-CSF) method. We focus on the particular situation of spreading drops. We first present the numerical methods used for the simulation of moving contact line i.e. static contact angle versus dynamic contact angle, no slip condition versus slip condition. A grid and time convergence is performed for the different models. We show that the integration of the Continuum Surface Force using the finite volume method results in a grid dependence at the onset of the spreading. The static and dynamic models are compared to experiments. It is shown that the dynamic models based on the Cox's relation for the dynamic contact angle are able to reproduce experiments while static models overestimate the spreading time and are not able to reproduce the Tanner regime. The difference between static and dynamic models is shown to increase with the Ohnesorge number.
\end{abstract}

Moving contact line

Numerical model

Spreading drop where $\Delta$ is the grid spacing and $\mu$ is the fluid viscosity. The stress at the contact line is clearly diverging when refining the grid size (see for example [14] where the evolution of the viscous stress at the wall is reported). Several authors $[8,6,14]$ have dealt with the "stress singularity" paradox by introducing the Navier slip condition that gives a relation between the fluid velocity at the wall $U_{W}$ and a Navier slip length $\lambda_{N}$ :

$U_{W}=\lambda_{N} \frac{\partial U}{\partial n_{W}}$

where $n_{W}$ is the normal to the wall. The grid convergence is then obtained by solving the full hydrodynamic problem inside the hydrodynamics slip region. Unfortunately, due to the grid refinement limitation, most of these simulations use unrealistically large slip length values and therefore the Navier slip length $\lambda_{N}$ becomes in practice an adjustable parameter for the simulation (see Bonn et al. [15]). The grid convergence of the simulations is then reached but an unphysical slip condition is necessary. In recent developments, the dynamic or apparent contact angle is connected to the velocity of the contact line. The Cox [16] relation is directly applied [10,17] or adapted using an adjustable parameter that needs to be empirically determined from experiments $[13,14]$.

As shown in this introduction, different strategies have been developed for the simulation of moving contact line. We discuss and compare different possible modelings in the first part of this paper. Then the numerical models are compared with experiments of spreading drop.
Corresponding author at: University of Toulouse, INPT-UPS,
Mécanique des Fluides de Toulouse, France. Tel.: +33 534322818 .

E-mail address: legendre@imft.fr (D. Legendre). 


\section{Numerical method}

\subsection{VoF solver}

The numerical simulations reported in this work are performed with the Volume of Fluid (VoF) solver developed in the JADIM code $[18,10]$. The one-fluid system of equation is obtained by introducing the one-fluid function $C$ used to localize one of the two phases. In this study, we define $C$ as $C=1$ in the liquid, here the drop, and $C=0$ in the external fluid. The one-fluid function $C$ makes possible the definition of the one fluid variables $U=C U_{1}+(1-C) U_{2}$ for the velocity, $P=C P_{1}+(1-C) P_{2}$ for the pressure, $\rho=C \rho_{1}+(1-C) \rho_{2}$ for the density and $\mu=C \mu_{1}+(1-C) \mu_{2}$ for the viscosity. The position of the interface is then given by the transport equation:

$\frac{\partial C}{\partial t}+U \cdot \nabla C=0$

The two fluids are assumed to be Newtonian and incompressible with no phase change. Under isothermal condition and in the absence of any surfactant the surface tension is constant and uniform at the interface between the two fluids. In such condition, the velocity field $U$ and the pressure $P$ satisfy the classical one-fluid formulation of the Navier-Stokes equations:

$\nabla \cdot U=0$

$\rho\left(\frac{\partial U}{\partial t}+U \cdot \nabla U\right)=-\nabla P+\nabla \cdot \Sigma+\rho g+F_{\sigma}$

where $\Sigma$ is the viscous stress tensor, $g$ is the gravity and $F_{\sigma}$ is the capillary contribution:

$F_{\sigma}=\sigma \nabla \cdot n n \delta_{I}$

where $\sigma$ is the surface tension, $n$ denotes by arbitrary choice the unit normal of the interface going out from the drop and $\delta_{I}$ is the Dirac distribution associated to the interface.

The system of Eqs. (3)-(6) is discretized using the finite volume method. Time advancement is achieved through a third-order Runge-Kutta method for the viscous stress. Incompressibility is satisfied at the end of each time step though a projection method. The overall algorithm is second-order accurate in both time and space. The volume fraction $C$ and the pressure $P$ are volumecentred and the velocity components are face-centred. Due to the discretization of $C$, it results a numerical thickness of the interface, cells cut by the interface corresponding to $0<C<1$. The interface location and stiffness are both controlled by an accurate transport algorithm based on FCT (Flux-Corrected-Transport) schemes [19]. This method leads to an interface thickness of about three grid cells by the implementation of a specific procedure for the velocity used to transport $C$ in flow region of strong strain and shear [18]. The interfacial force is solved using the classical CSF (Continuum Surface Force) model [20]:

$F_{\sigma}=\sigma \nabla \cdot\left(\frac{\nabla C}{|\nabla C|}\right) \nabla C$

The induced spurious currents have been characterized [10] and their maximum magnitude evolve as $0.004 \sigma / \mu$, in agreement with other codes using the Brackbill's formulation.

\subsection{Numerical modeling of the contact angle}

The numerical method for the simulation of static and dynamic contact angles has been developed by Dupont and Legendre [10] for 2D and axisymmetric geometries, and recently extended to $3 \mathrm{D}$ geometries [21]. The calculation of the capillary term requires the knowledge of the value of the contact angle made by the interface at the wall. Indeed, the capillary contribution in the momentum Eq. (6) requires the knowledge of $\nabla C$. Furthermore, $\nabla C /|\nabla C|$ being the normal of the interface, the boundary condition for $\nabla C$ is thus directly given by the value of the contact angle $\theta_{W}$ by the following relation:

$\frac{\nabla C}{|\nabla C|}=n=\sin \theta_{W} n_{\|}+\cos \theta_{W} n_{\perp}$

where the unit vectors $n_{\|}$and $n_{\perp}$ are the components of the normal vector $n$, parallel and normal to the wall, respectively. The general method is decomposed into two steps. We first determine the value of the contact angle to apply at the wall. This value is then imposed as a boundary condition using relation (8) for the calculation of the capillary contribution (7) in the momentum balance (5). One objective of this work is to compare different possible modeling to the dynamic modeling introduced in our code JADIM (model Dyn2 in the following). The tested models are reported in Table 1. The two main parameters that characterize these models are the description of the contact angle $\theta_{W}(t)$ and the description of the fluid boundary condition. The Navier slip condition (1) can be imposed in order to remove the stress singularity at the contact line with the introduction of the Navier slip length $\lambda_{N}$. If $\lambda_{N}=0$ a classical no-slip condition is imposed. When considering ordinary fluids and wall properties, a relevant value for the slip length is $\lambda_{N}=O\left(10^{-9}\right) \mathrm{m}$ [22]. Note that imposing such slip lengths for solving millimeter size drop with 100 regular cells per radius $\left(\Delta \sim R / 100 \sim 10^{-5} \mathrm{~m}\right)$ which is a very accurate description of the macroscopic flow field is equivalent to impose a no-slip condition. In the following we consider two sorts of model: "static" models (Stat1, Stat2 and Stat3) and "dynamic" models (Dyn1, Dyn2, Dyn3 and Dyn4). The simplest model, called "Stat1", consists in imposing the contact angle constant as the Young value $\theta_{S}$ with no slip condition $\lambda_{N}=0$. When imposing a constant contact angle $\theta_{W}(t)=\theta_{S}$, the effect of the sliding condition (1) has been examined by imposing a slip length linked to the grid size $\lambda_{N}=\Delta / 2$ (model "Stat2") as suggested by Afkhami et al. [14] or a fixed value for the slip length $\lambda_{N}=\Delta_{32} / 2$ (model "Stat3") where $\Delta_{32}$ is the grid spacing corresponding to 32 regular cells per radius which is the coarser grid used in this study (see next section for the description of the numerical parameters). The dynamic models are expressed as a function of the contact line Capillary number Ca defined as

$\mathrm{Ca}=\frac{\mu_{1} U_{c l}}{\sigma}$

where $U_{c l}$ is the contact line velocity. In our VoF formulation, $U_{c l}$ is the interface velocity interpolated at $C=0.5$. Due to the staggered grid structure, $U_{c l}$ is located at the distance $\Delta / 2$ from the wall where the node of the tangential component of the velocity that transports the interface is located. We have first tested the model (called "Dyn4") proposed by Afkhami et al. [14]. Based on 2D simulations and the expression developed by Cox [16], they proposed the following expression for the dynamic contact angle

$\cos \theta_{d}=\cos \theta_{S}+5.63 \mathrm{Ca} \log \left(\frac{K}{\Delta / 2}\right)$

The simulations reported by Afkhami et al. [14] show that it ensures grid convergence in VoF simulations when coupled with a slip length based on the grid spacing $\lambda_{N}=\Delta / 2$. The authors suggest that "the true value of $K$ could be determined by fitting numerical data to data obtained experimentally". In their simulations, a constant value $K=0.2 L$ is proposed for a plate withdrawing from a square fluid pool of length $L$ while the authors use $K=0.04 R$ for their simulations of the spreading of droplet of initial radius $R$. This smaller value of $K$ was chosen because their model is only valid for $\left|\cos \theta_{d}\right|<0.6$. The model "Dyn1" corresponds to the original model implemented in JADIM by Dupont and Legendre [10]. The dynamic 
Table 1

Numerical model considered for the dynamic contact line. $\theta_{W}$ is the contact angle imposed on the wall and $\lambda_{N}$ is the slip length.

\begin{tabular}{|c|c|c|c|}
\hline Name & Contact angle & Navier condition & Reference \\
\hline Stat1 & $\theta_{W}=\theta_{S}$ & $\lambda_{N}=0$ & \\
\hline Stat2 & $\theta_{W}=\theta_{S}$ & $\lambda_{N}=\Delta / 2$ & \\
\hline Stat3 & $\theta_{W}=\theta_{S}$ & $\lambda_{N}=\Delta_{32} / 2$ & \\
\hline Dyn1 & $\theta_{W}=\theta_{d}$ (Eq. (11)) with $L=10^{-6}, \lambda=10^{-9} \mathrm{~m}$ & $\lambda_{N}=0$ & {$[10]$} \\
\hline Dyn2 & $\theta_{W}=\theta_{d}\left(\right.$ Eq. (11)) with $L=\Delta / 2, \lambda=10^{-9} \mathrm{~m}$ & $\lambda_{N}=0$ & {$[23,21,17]$} \\
\hline Dyn3 & $\theta_{W}=\theta_{d}$ (Eq. (11)) with $L=\Delta / 2, \lambda=10^{-9} \mathrm{~m}$ & $\lambda_{N}=\Delta / 2$ & \\
\hline Dyn4 & $\theta_{W}=\theta_{d}($ Eq. $(10))$ & $\lambda_{N}=\Delta / 2$ & {$[14]$} \\
\hline
\end{tabular}

contact angle is given by the hydrodynamic model derived by Cox [16]:

$g\left(\theta_{d}\right)=g\left(\theta_{S}\right)+$ Ca $\log \left(\frac{L}{\lambda}\right)$

where $g(\theta)$ is a function that simplifies to:

$g(\theta)=\int_{0}^{\theta} \frac{x-\sin x \cos x}{2 \sin x} d x$

when the surrounding fluid is of much smaller viscosity (for example air). In practice the function $g$ and $g^{-1}$ can be approximated with a good accuracy with fitting polynomial [10]. Note that, when the condition $\theta_{d}<3 \pi / 4$ is satisfied, a simplified form of Eq. (11) is the well known relation $\theta_{d}^{3}=\theta_{S}^{3}+C a \log (L / \lambda)$. In the model "Dyn1" [10] the apparent length $L$ is imposed to be $L=10 \mu \mathrm{m}$ and the slip length is $\lambda=10^{-9} \mathrm{~m}$. The grid spacing $\Delta$ is then chosen of the same order as the apparent length $\Delta \approx L$. In the model "Dyn2" used in $[23,21,17]$, we impose the apparent length $L$ as the location of the first node of the VoF function: $L=\Delta / 2$. The model "Dyn3" is the model "Dyn2" combined with the slip condition (1) using a numerical slip length $\lambda_{N}=\Delta / 2$.

\section{Time and grid convergences}

\subsection{Test case presentation}

For the time and grid convergence discussion as well as for the comparison between the models, we first consider the test case reported by Afkhami et al. [14]. Note that the simulations reported by Afkhami et al. [14] are performed for a semicircular droplet (2D simulation) while we consider here a 3D drop thanks to an axisymmetric simulation. The tests reported here have also been performed for a 2D drop and lead to the same conclusion. We consider a spherical cap droplet of radius $R_{0}$ at equilibrium on an horizontal wall so that the contact angle made by the initial shape is $\theta_{i}=90^{\circ}$ (Fig. 1a). No gravity effect is considered so that we impose
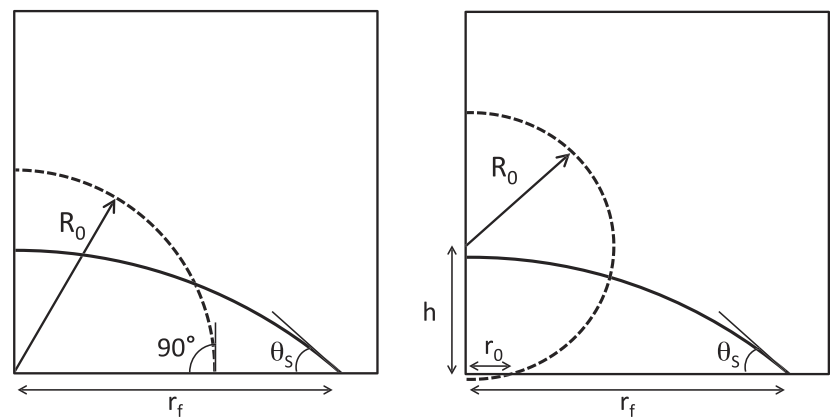

Fig. 1. Initial drop shape (dashed line) and final drop shape (base radius $r_{f}$ ) for the two cases considered. (a) For the comparison between the models (test case proposed by Afkhami et al. [14]). (b) For the comparison with the experiments where $r_{0}$ and $h$ are the initial base radius and the initial distance between the drop centre and the wall, respectively. $g=0 \mathrm{~m} / \mathrm{s}^{-2}$. The computational domain is a square domain of equal radial and vertical extension $L_{r}=L_{z}=2 R_{0}$. The axis of symmetry corresponds to the west boundary, the wetting conditions given by Table 1 (slip and contact angle) are imposed on the south boundary and classical wall conditions (no slip) are imposed on the two other boundaries (east and north). The value of the contact angle made by the contact line is initially $\theta_{i}=90^{\circ}$ and the drop spreads until the drop stabilizes with a spherical cap shape satisfying the Young contact angle $\theta_{s}$. The value of the parameters used for the simulation are $\rho_{1}=\rho_{2}=1, \mu_{1}=\mu_{2}=0.25, \sigma=7.5$ and $\theta_{S}=60^{\circ}$ [14]. The reported results are shown using normalized quantities. The radial position of the interface is normalized by $R_{0}$ and the normalized time is $\tau=t \mu / \rho R_{0}^{2}$.

\subsection{Numerical parameters}

The time steps used for the time convergence are listed in Table 2. The grids used for the grid convergence are presented in Table 3. The numbers of node in each direction are noted $N_{r}$ and $N_{z}$ respectively. Four grids, $M_{32}, M_{64}, M_{128}$ and $M_{256}$, are considered corresponding to $32,64,128$ and 256 nodes in each direction, respectively.

\subsection{Time and grid convergence}

We have first considered the time convergence for both the static and dynamic models. The time evolution of the base radius $r(t)$ and the Capillary number $\mathrm{Ca}=\mu U_{c l} / \sigma$ as well as the velocity gradient $d U / d y$ on the wall have been inspected. For all the grids and models considered a time convergence of the simulation can be reached. We have observed that the corresponding value of the time step ensuring time convergence significantly decreases with the grid refinement. As a consequence, the time steps $\Delta t_{5}=5 \times 10^{-5}, \Delta t_{7}=10^{-5}, \Delta t_{8}=5 \times 10^{-6}$ and $\Delta t_{9}=2 \times 10^{-6}$ are respectively chosen for the grids $M_{32}(32 \times 32), \quad M_{64}$

Table 2

Time step values used in the simulations. Depending on the case under consideration the value are non-dimensional or dimensional (expressed in second).

\begin{tabular}{lllllll} 
Name & $\Delta t_{1}$ & $\Delta t_{2}$ & $\Delta t_{3}$ & $\Delta t_{4}$ & $\Delta t_{5}$ & $\Delta t_{6}$ \\
Value & $10^{-3}$ & $5 \times 10^{-4}$ & $2 \times 10^{-4}$ & $10^{-4}$ & $5 \times 10^{-5}$ & $2 \times 10^{-5}$ \\
$\Delta t_{7}$ & $\Delta t_{8}$ & $\Delta t_{9}$ & $\Delta t_{10}$ & $\Delta t_{11}$ & $\Delta t_{12}$ & $\Delta t_{13}$ \\
$10^{-5}$ & $5 \times 10^{-6}$ & $2 \times 10^{-6}$ & $10^{-6}$ & $5 \times 10^{-7}$ & $2 \times 10^{-7}$ & $10^{-7}$ \\
\hline
\end{tabular}

Table 3

Grid used in the simulations reported in this section.

\begin{tabular}{lllll}
\hline Name & $\Delta$ & $L_{r}$ & $L_{z}$ & $N_{r} \times N_{z}$ \\
\hline$M_{32}$ & $1 / 32$ & 1 & 1 & $32 \times 32$ \\
$M_{64}$ & $1 / 64$ & 1 & 1 & $64 \times 64$ \\
$M_{128}$ & $1 / 128$ & 1 & 1 & $128 \times 128$ \\
$M_{256}$ & $1 / 256$ & 1 & 1 & $256 \times 256$ \\
\hline
\end{tabular}


$(64 \times 64), M_{128}(128 \times 128), M_{256}(256 \times 256)$ in the simulations reported for the grid convergence.

Considering now the grid convergence, we first focus on the "static" models (see Table 1). The contact angle is set fixed to the Young angle $\theta_{S}$ and the models differ by the slip length condition used in the Navier condition. The slip conditions are $\lambda_{N}=0, \lambda_{N}=\Delta / 2$ and $\lambda_{N}=\Delta_{32}=2 R_{0} / 32$ for the models Stat 1 , Stat2 and Stat3, respectively. The grid convergence is shown in Fig. 2 for the radius of the wetted surface and in Fig. 3 for the Capillary number and the velocity gradient at the wall.

The use of a Navier slip length clearly changes the drop spreading. The only way to observe a convergence with the grid (i.e. a
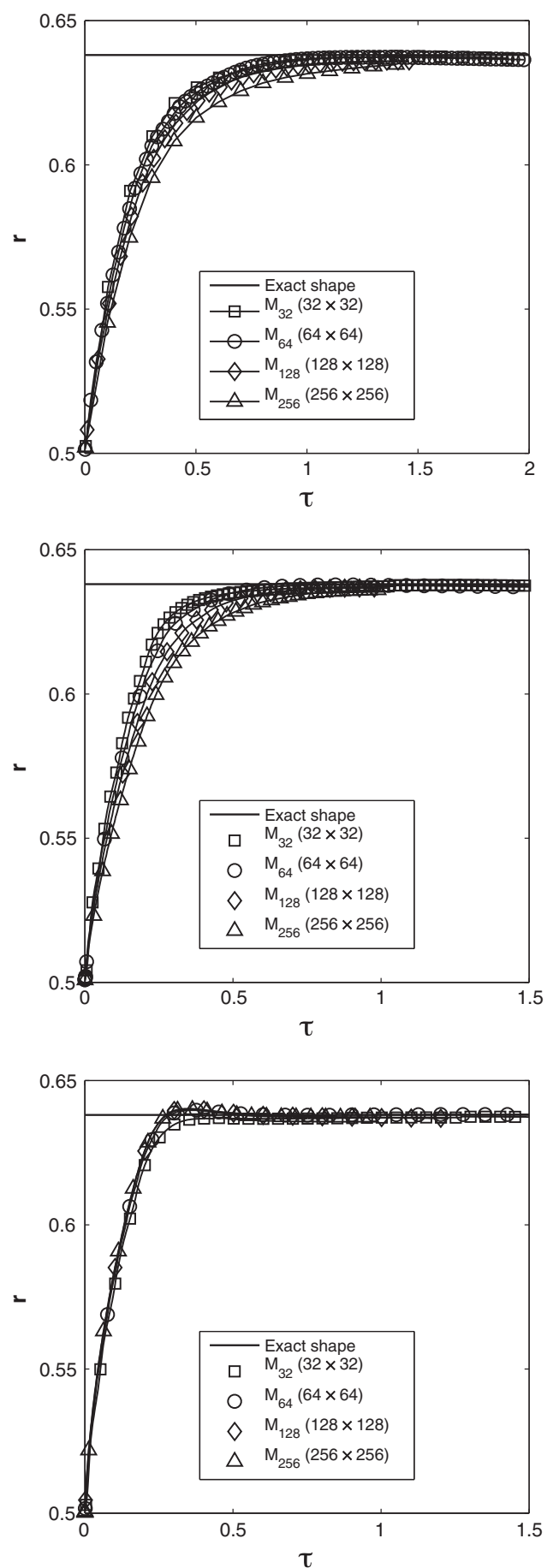

result that tends to be independent on the grid spacing when refining the grid) is to introduce a slip length larger than the grid size in order to solve the slip region. For all the models, the initial stage of the spreading is found to be very dependent on the grid as revealed by the evolution of the Capillary number. This point is discussed in detail in the next section. For this purpose a log-log representation is used in order to show the evolution of the contact line velocity from the beginning of the spreading, since one has $\mathrm{Ca}=0$ at $t=0$.

The models based on a dynamic contact angle are now considered. We first consider the model Dyn4 proposed by [14] in order to make their contact line simulations grid independent. The effect of the grid spacing shown in Fig. 4 reveals that this model is not
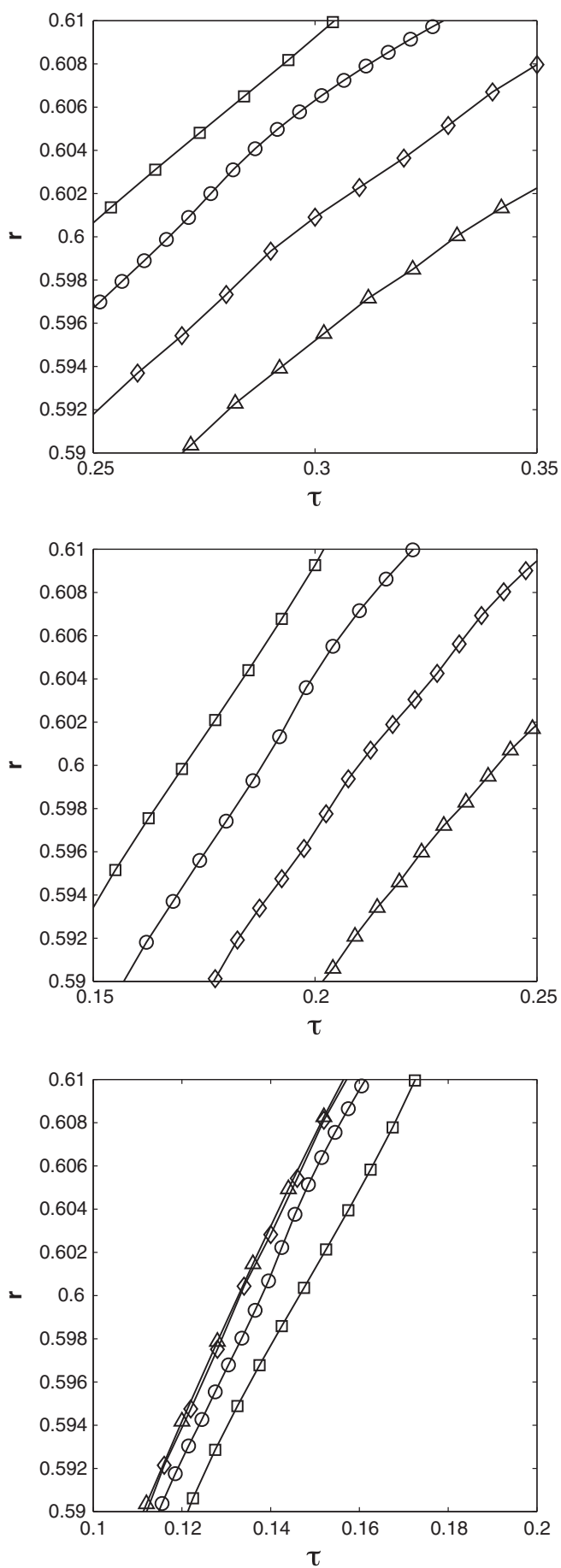

Fig. 2. Grid convergence for the 3 models using a static contact angle. From top to bottom: Stat1, Stat2 and Stat3. $\square M_{32}(32 \times 32)$, $\circ M_{64}(64 \times 64), \diamond M_{128}(128 \times 128)$, and $\triangle M_{256}(256 \times 256)$. 

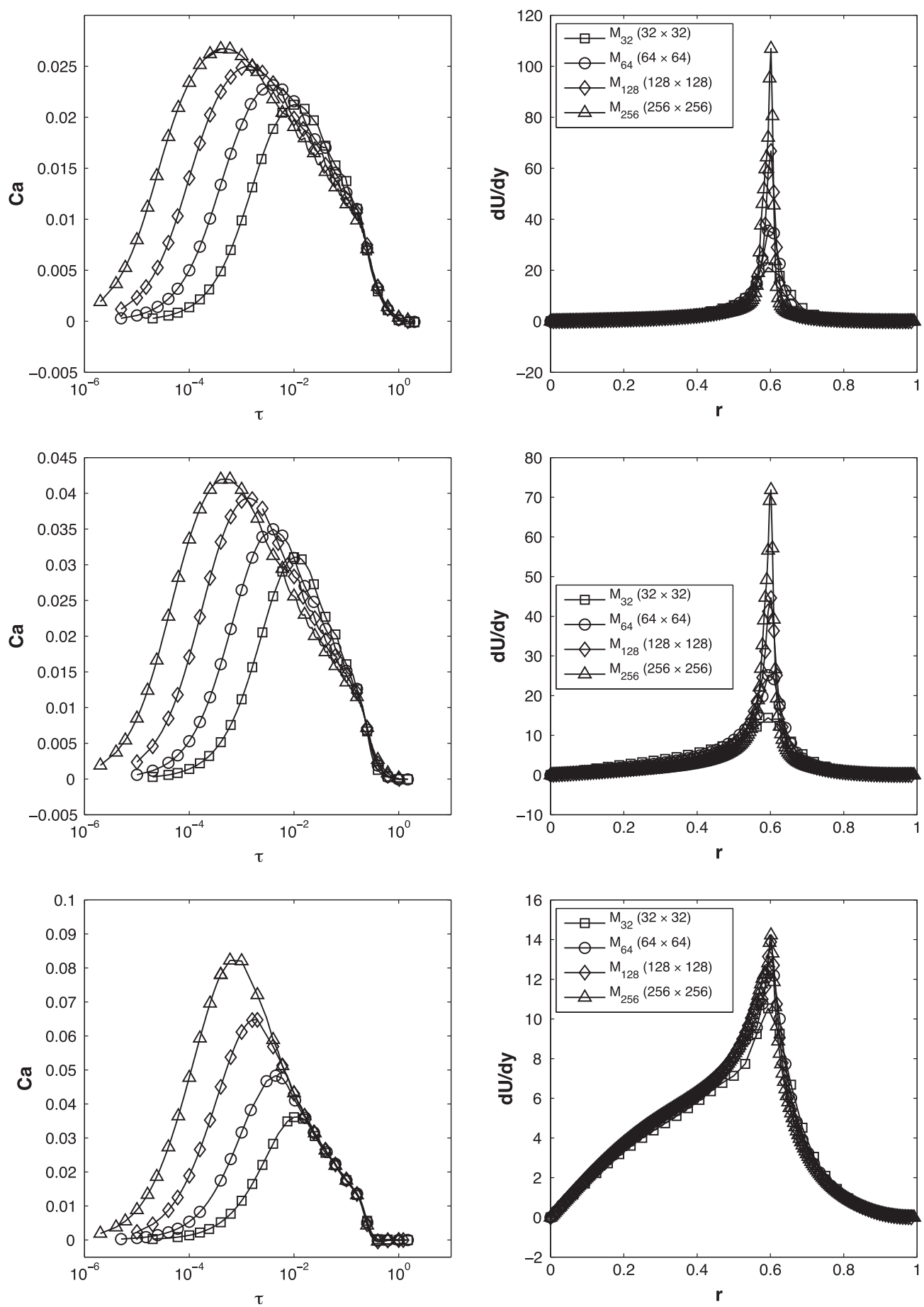

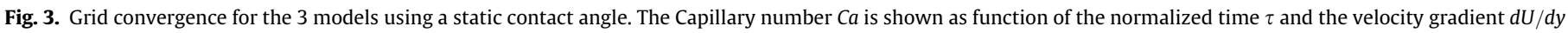
is shown for $r=0.6$. From top to bottom: Stat1, Stat2 and Stat3. $\square M_{32}(32 \times 32)$, 。 $M_{64}(64 \times 64), \diamond M_{128}(128 \times 128)$, and $\triangle M_{256}(256 \times 256)$.

grid independent when introduced in our code. The evolution of the contact angle have some unexpected evolutions very dependent on the grid spacing.

We finally consider the dynamic models Dyn1, Dyn2 and Dyn3 based on the dynamic angle expression (11). They differ by the apparent length $L$ : it is an imposed value based on physical observations $L=10 \mu \mathrm{m}$ for Dyn1 while it is the grid spacing $L=\Delta / 2$ for Dyn2 and Dyn3. The effect of the grid spacing on these model is shown in Fig. 5 for the base radius $r(t)$ and in Fig. 6 for the Capillary number and the velocity gradient at the wall. Strictly speaking, grid convergence is not reached (this point will be discussed in more details the last section) but it is clearly improved compared to the static models. We observe that the introduction of a slip length on the wall based on the grid spacing (model Dyn3) do not improve significantly the grid convergence and the two models Dyn2 and Dyn3 give a very similar behavior. Note that we have also tested a slip condition just located on the surface in contact with the interface $(0.05 \leqslant C \leq 0.95)$. The results obtained with this model are very similar to the results obtained with the model Dyn3.

For all the models considered, the evolution of the Capillary number reveals an important effect of the grid spacing at the beginning of the spreading, typically for $\tau<10^{-2}$. This particular behavior is inspected in the next section. 

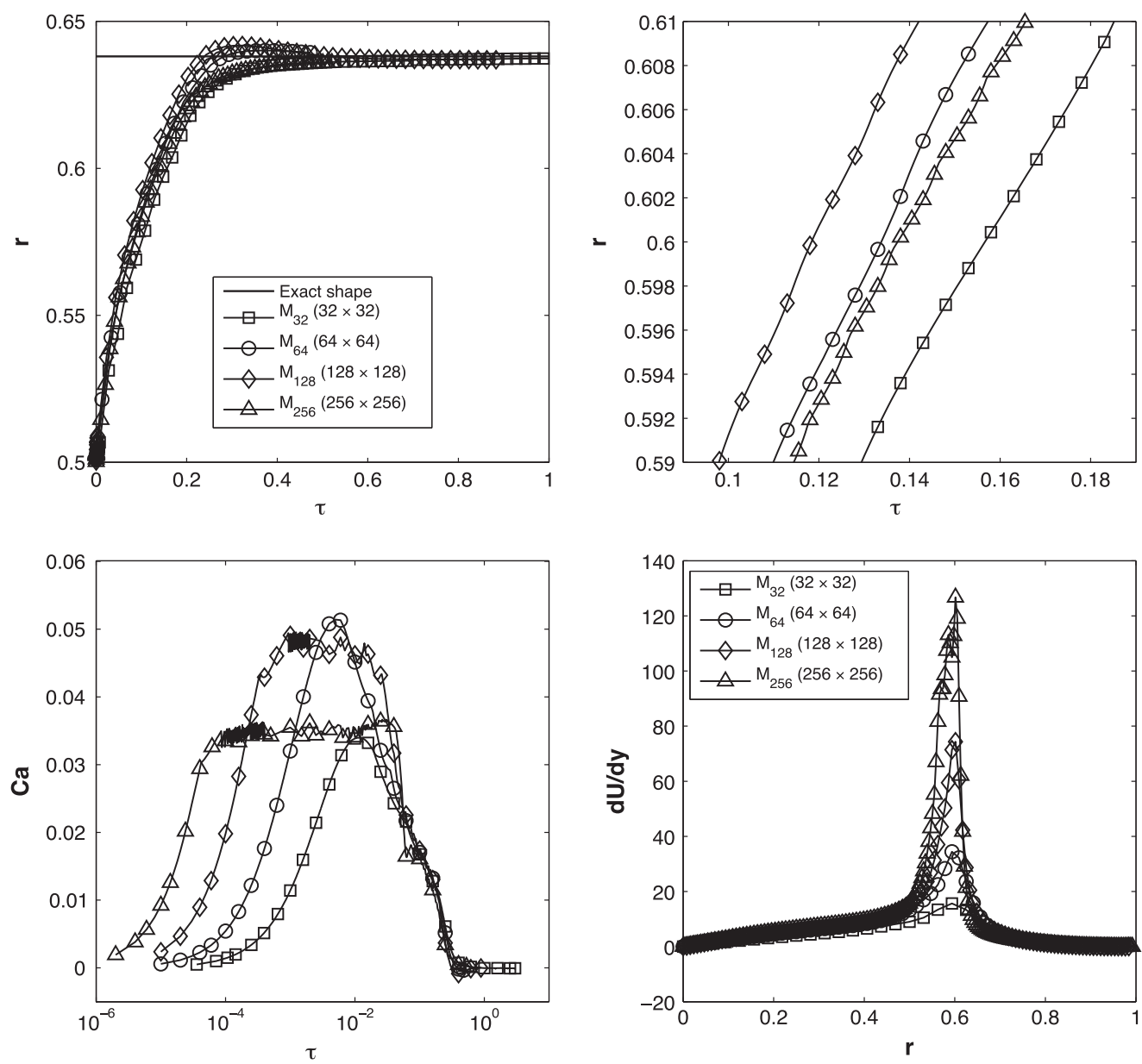

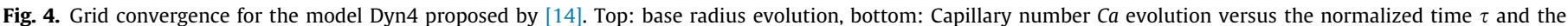
velocity gradient $d U / d y$ reported at $r=0.6$. $\square M_{32}(32 \times 32)$, $\circ M_{64}(64 \times 64), \diamond M_{128}(128 \times 128)$, and $\Delta M_{256}(256 \times 256)$.

\section{Comparison between models}

In this section we compare the different models. We first focus on the contact line radial evolution and then a special attention is made on the initial acceleration of the contact line.

The time evolution of the radial position $r(t)$ of the contact line is reported in Fig. 7 for the grid $M_{64}(64 \times 64)$ and $\Delta t_{7}=10^{-5}$. This figure clearly shows a significant difference between the models. The spreading times differ by almost one order of magnitude between static and dynamic models. The "static" models show a faster drop spreading. The model proposed by [14] is found to give similar evolution as that given by the "static" models.

As commented before, the initial contact line velocity appears to be very sensible to the grid spacing. The comparison between 3 different models (Stat1, Stat3 and Dyn2) in reported in Fig. 8 for the four grids $M_{32}, M_{64}, M_{128}$ and $M_{256}$. It is shown that the initial velocity is independent on the contact angle model but it is clearly imposed by the grid spacing. The evolution is linear indicating an initial constant acceleration which is found to increase when refining the grid.

At equilibrium, the Laplace pressure jump controls the shape of the drop:

$-\nabla P_{0}+F_{\sigma 0}=0$

where the surface tension contribution is

$F_{\sigma 0}=\sigma \operatorname{div}\left(n_{I}\right) n_{I} \delta_{I}$
Following the CSF formulation for $F_{\sigma}$, the normal of the interface is calculated as $n_{I}=\frac{\nabla C}{\|\nabla C\|}$ and the Dirac term is $n_{I} \delta_{I}=\nabla C$. Thus considering the finite volume method used for the discretization of the equations, the surface tension contribution integrated in a control volume $V$ is expressed as

$F_{\sigma 0} V=\sigma \int_{V} \operatorname{div} \frac{\nabla C}{\|\nabla C\|} d V \nabla C$

where the divergence term corresponding to the curvature is converted to a surface integral. This term is then evaluated as fluxes of the interface normal $n_{I}=\frac{\nabla C}{\|\nabla C\|}$ at the surface $\Sigma$ of each control volume:

$F_{\sigma 0} V=\sigma \int_{\Sigma} n_{I} \cdot n_{\Sigma} d \Sigma \nabla C$

Considering the control volume containing the contact line, the south contribution of the normal is given by the contact angle $\theta_{W}$ :

$n_{I}^{S}=-\cos \theta_{W}$

When changing the contact angle from $\theta_{W}^{0}$ to $\theta_{W}$ at $t=0$, the momentum equation becomes

$\rho\left(\frac{\delta u}{\delta t}\right)_{t=0^{+}}=-\nabla P^{0}+F_{\sigma}$

Considering relations (13), (16) and (17), the integration of Eq. (18) on a control volume $V$ gives the initial acceleration $a^{0}$ : 

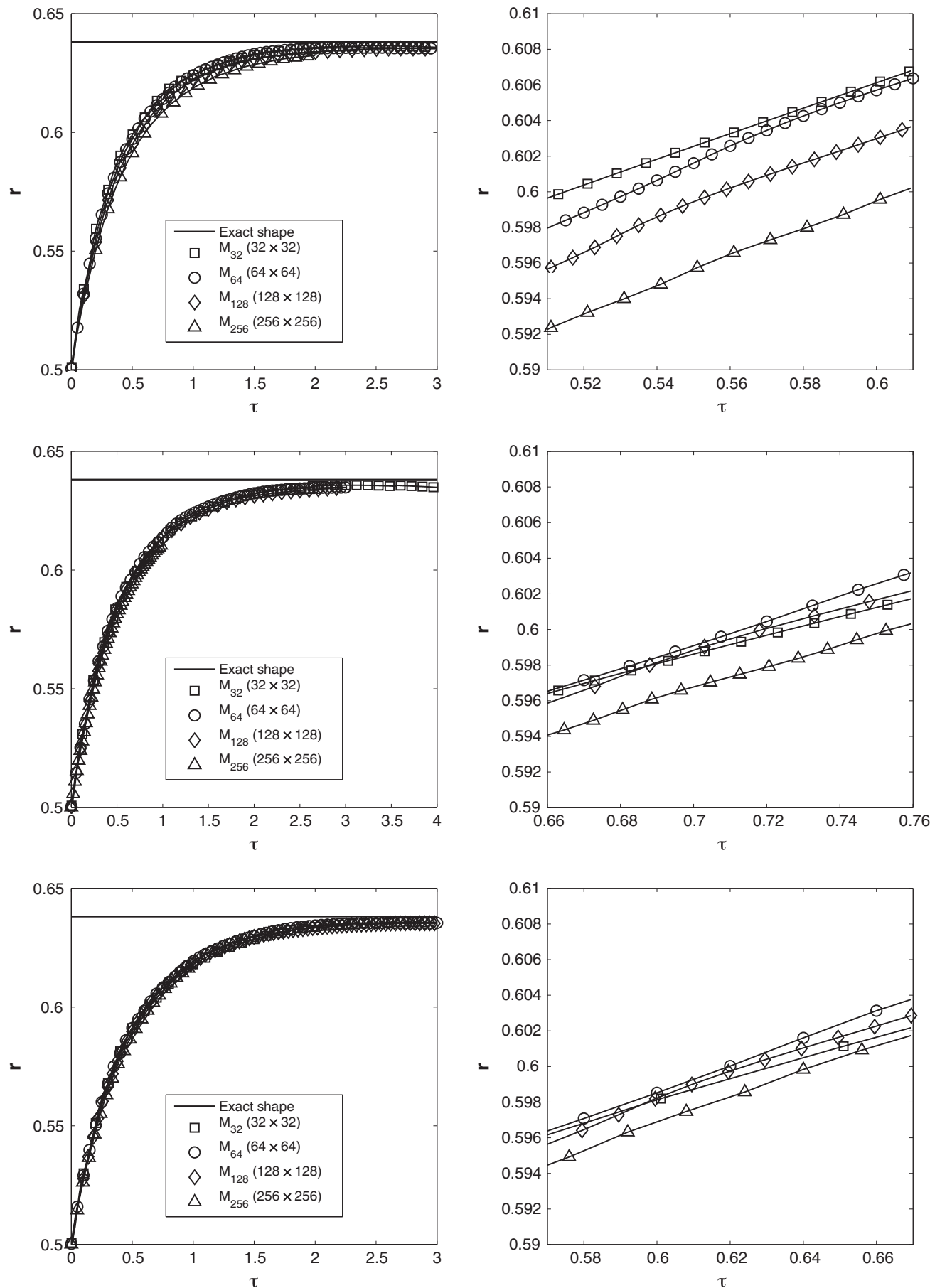

Fig. 5. Grid convergence for the dynamic models. Evolution of the radial evolution of the wetted area. From top to bottom: Dyn1, Dyn2, Dyn3. $\square M_{32}\left(32 \times 32\right.$ ), $\circ M_{64}$ $(64 \times 64), \diamond M_{128}(128 \times 128)$, and $\triangle M_{256}(256 \times 256)$.

$a^{0}=\left(\frac{\delta u}{\delta t}\right)_{t=0^{+}}=\frac{\sigma}{\rho}\left(\cos \theta_{W}-\cos \theta_{W}^{0}\right) \frac{\Sigma^{S}}{V} \nabla C$

where $\Sigma^{S}$ is the south surface of the control volume. For the axisymmetric problem under consideration, the control volume containing the interface located at $r=R_{c}$ is characterized by $V=2 \pi R_{c} \Delta^{2}$ and $\Sigma=2 \pi R_{c} \Delta$ while the Dirac term projected on the radial direction is calculated as

$\nabla C . e_{r}=\frac{R_{c}-\Delta / 2}{R_{c}} \frac{1-0}{\Delta} \sim \frac{1}{\Delta}$

Finally the radial acceleration of the contact line resulting from the finite volume discretization of the momentum equation is $a_{r}^{0} \sim \frac{\sigma}{\rho}\left(\cos \theta_{W}-\cos \theta_{W}^{0}\right) \frac{1}{\Delta^{2}}$

showing a grid dependence of order -2 . Fig. 9 reports the initial acceleration calculated for the four grids and for different contact angles $\theta_{W}$, surface tensions $\sigma$ and densities $\rho$. The $\log -\log$ representation confirms that the initial acceleration of the contact line evolves as $\sigma \cos \theta_{W} / \rho \Delta^{2}$ since $\theta_{W}^{0}=90^{\circ}$.

\section{Spreading drop}

The objective is now to compare the models with experiments of spreading drop. In the experiments, a drop of volume 

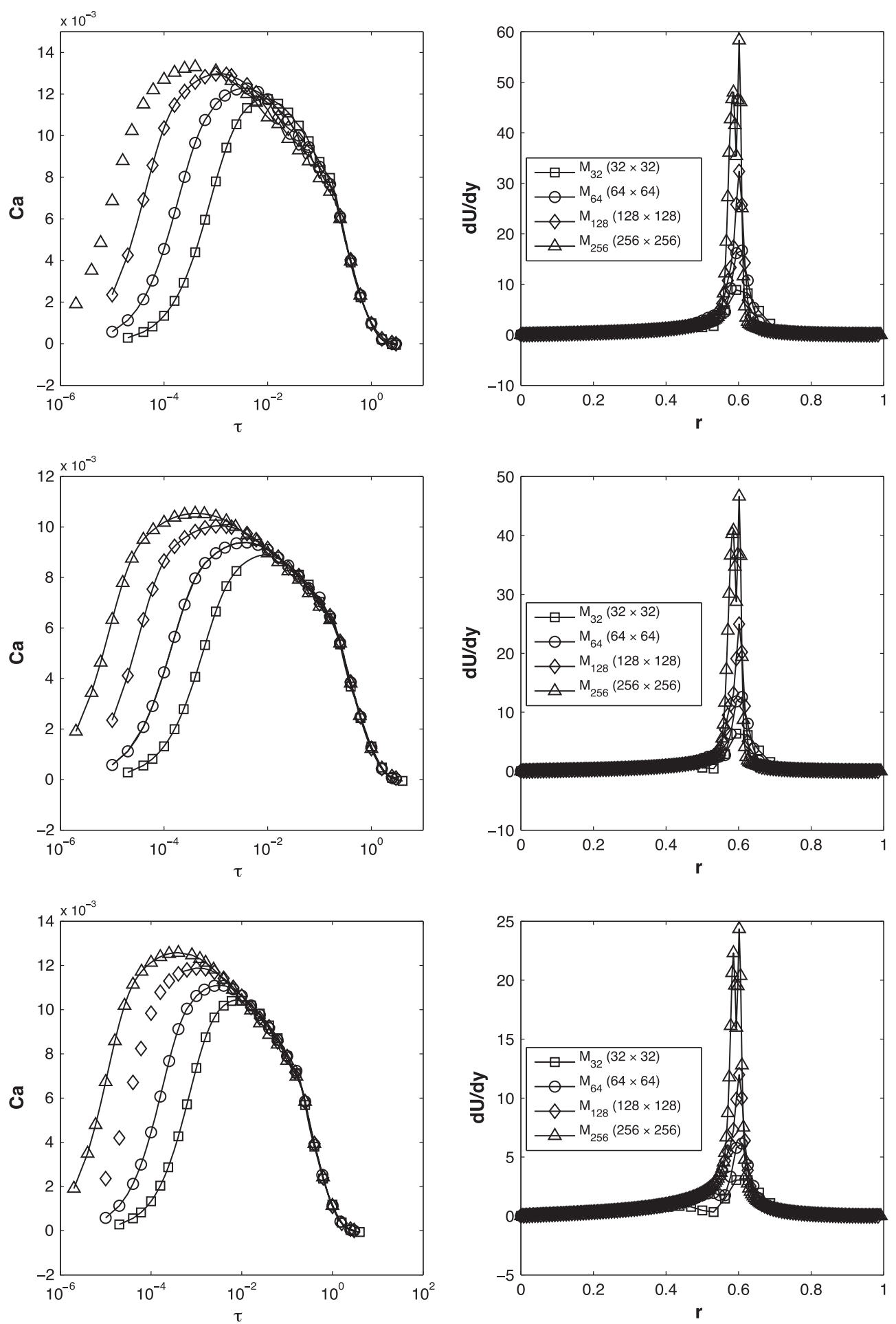

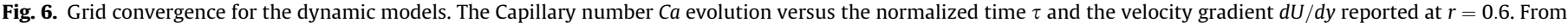
top to bottom: Dyn1, Dyn2, Dyn3. $\square M_{32}(32 \times 32), \circ M_{64}(64 \times 64), \diamond M_{128}(128 \times 128)$, and $\triangle M_{256}(256 \times 256)$.

$V=4 \pi R_{0}^{3} / 3$ is deposited on a wall with no initial velocity (see Fig. 1b). Squalane and water drops are considered for comparison with the experiments of Lavi and Marmur [24], Winkels et al. [25], respectively. The corresponding Ohnesorge numbers $O h=\mu / \sqrt{\rho \sigma R_{0}}$ are $O h=0.21$ and $O h=0.0053$, respectively.

As shown in Fig. 1b, the drop centre is initially located at the distance $h \leqslant R_{0}$ from the wall. The effect of this initial condition has been analyzed by considering different initial locations (see Table 4).
The characteristics of the grids used for the simulations are given in Table 5. Axisymmetric simulations have been performed in a grid of radial extension $L_{r}=3 R_{0}$ and vertical dimension $L_{z}=3 R_{0}$. The numbers of nodes in each direction are noted $N_{r}$ and $N_{z}$ respectively. Different grids are considered corresponding to $32,64,128$ and 167 nodes per radius, respectively.

We have first performed some simulations corresponding to the experiments of Lavi and Marmur [24] for millimeter squalane drops, a fluid 30 times more viscous than water. The physical 


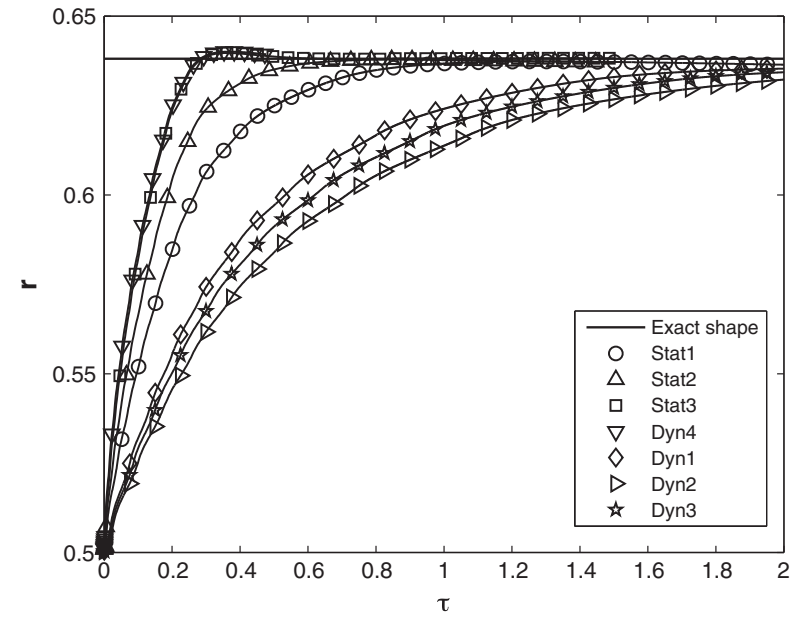

Fig. 7. Comparison between the different models for $M_{64}(64 \times 64)$ and $\Delta t_{7}=10^{-5}$.

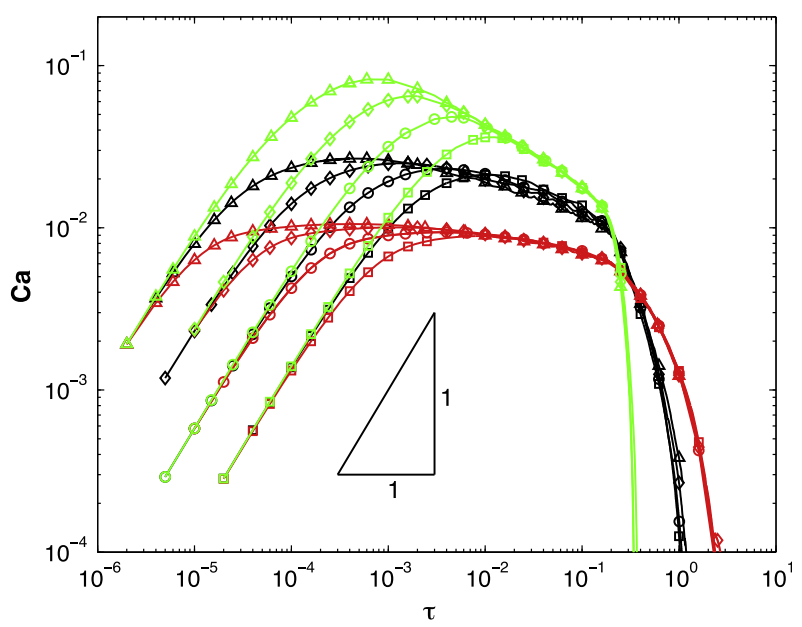

Fig. 8. Evolution of the Capillary number for the different grids and for the models: Stat1 (black), Stat3 (green) and Dyn2 (red). $\square M_{32}(32 \times 32)$, 。 $M_{64}(64 \times 64), \diamond M_{128}$ $(128 \times 128)$, and $\triangle M_{256}(256 \times 256)$. (For interpretation of the references to colour in this figure legend, the reader is referred to the web version of this article.)

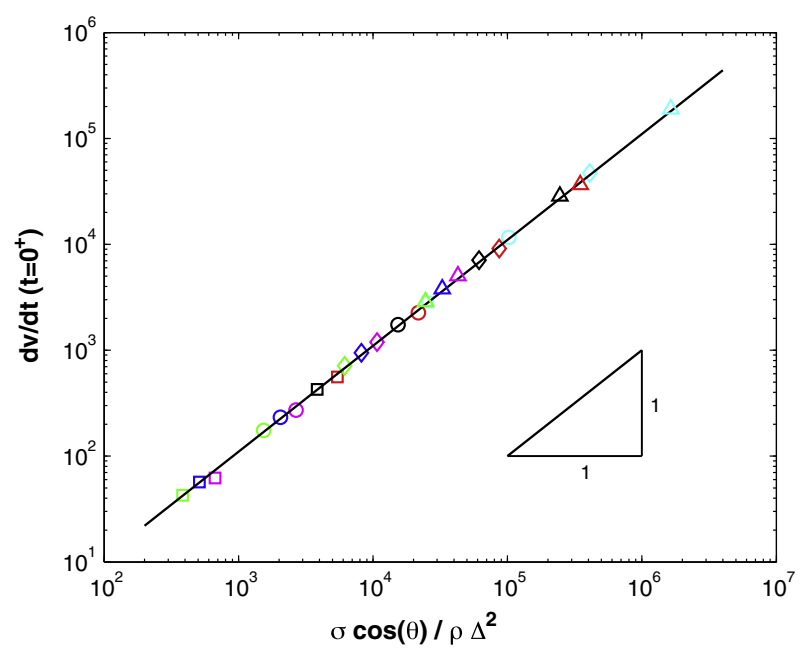

Fig. 9. Initial acceleration of the contact line. $\square M_{32}(32 \times 32)$, $\circ M_{64}(64 \times 64), \diamond$ $M_{128}(128 \times 128), \Delta M_{256}(256 \times 256)$. For $\theta_{S}=60^{\circ}($ dark $), \theta_{S}=75^{\circ}($ red $), \theta_{S}=45^{\circ}$ (blue), $\theta_{S}=80^{\circ}$ (magenta), $\theta_{S}=85^{\circ}$ (cyan), and $\theta_{S}=67^{\circ}$ (dark). (For interpretation of the references to color in this figure legend, the reader is referred to the web version of this article.)
Table 4

Initial distance between the drop centre and the wall.

\begin{tabular}{llllll}
\hline Name & $h_{1}$ & $h_{2}$ & $h_{3}$ & $h_{4}$ & $h_{5}$ \\
Value & $0.95 R_{0}$ & $0.98 R_{0}$ & $0.99 R_{0}$ & $0.995 R_{0}$ & $0.998 R_{0}$ \\
\hline
\end{tabular}

Table 5

Grid used in the simulations reported in Section 5.

\begin{tabular}{lllll}
\hline Name & $\Delta / R_{0}$ & $L_{r} / R_{0}$ & $L_{z} / R_{0}$ & $N_{r} \times N_{z}$ \\
\hline$M_{96}$ & $1 / 32$ & 3 & 3 & $96 \times 96$ \\
$M_{192}$ & $1 / 64$ & 3 & 3 & $192 \times 192$ \\
$M_{384}$ & $1 / 128$ & 3 & 3 & $384 \times 284$ \\
$M_{500}$ & $3 / 500$ & 3 & 3 & $500 \times 500$ \\
\hline
\end{tabular}

properties of of the squalane used in the experiments are $\rho=809 \mathrm{~kg} / \mathrm{m}^{3}, \mu=0.034 \mathrm{~Pa} \mathrm{~s}, \sigma=0.032 \mathrm{~N} / \mathrm{m}, R_{0}=1 \mathrm{~mm}$ and the value of the static contact angle is $\theta_{S}=41.5^{\circ}$. The grid and time convergences have been reported in Dupont and Legendre [10] for both the models Stat1 and Dyn1. The effect of the time step and grid spacing are shown for the model Dyn2 in Fig. 10a and b, respectively. Both figures show that the difference is not perceptible when changing the time step and the grid spacing so that the time step $\Delta t_{8}$ and the grid $M_{192}$ are used in the following.

The effect of the initial position is shown in Fig. 11 by comparing three initial positions: $h_{1}=0.95 R_{0}, h_{2}=0.98 R_{0}$ and $h_{3}=0.99 R_{0}$. A significant difference is observed at the beginning of the spreading for the evolution of the base radius $r$ (see Fig. 12a) but after the time $t \sim 1 \mathrm{~ms}$, the same evolution is observed. Considering the initial radius $r_{0}$ and the final radius $r_{f}$ of the contact line, the normalized radius $r *=\left(r-r_{0}\right) /\left(r_{f}-r_{0}\right)$ is shown in Fig. 11b. Interestingly, all the evolutions are now very close, showing that the effect of the initial wetted area has not a significant impact on the drop spreading.

The simulations using the different models are now compared with the experimental data in Fig. 12. The normalized radius $r^{*}=\left(r-r_{0}\right) /\left(r_{f}-r_{0}\right)$ is reported versus the time normalized using the viscous-capillary time $t_{\mu}=\sigma / \mu V^{1 / 3}$. The three dynamic model Dyn1, Dyn2 and Dyn3 give very similar evolutions in agreement with the experiments while a significant difference is observed when using static contact angle models. As clearly shown, the choice of the contact angle model has a significant effect on the drop spreading.

We now consider the spreading of a water drop. We compare our simulations using the different models with the experiments performed by Winkels et al. [25]. Using high-speed imaging of experiments on millimeter-sized water drop in air, they have shown that the spreading regime is characterized by the power law $r \approx t^{1 / 2}$ consistent with a balance between the capillary pressure $\sim \sigma R_{0} / r^{2}$ and the inertial pressure $\sim \rho(d r / d t)^{2}$ at the contact line. The physical properties are $\rho=1000 \mathrm{~kg} / \mathrm{m}^{3}, \mu=0.001 \mathrm{~Pa} \mathrm{~s}$, $\sigma=0.0072 \mathrm{~N} / \mathrm{m}, R_{0}=0.5 \mathrm{~mm}$ and $\theta_{S}=115^{\circ}$. The effect of the time step and the grid spacing as well as the initial position of the drop have also been checked. Based on these tests, the simulations have been performed with the time step $\Delta t_{13}=10^{-7} \mathrm{~s}$, the grid $M_{384}$ and the initial position $h_{5}=0.998 R_{0}$.

The comparison with the experiments performed by Winkels et al. [25] is shown in Fig. 13. The agreement is found to be very satisfactory and the power law $t^{1 / 2}$ seems to be reproduced by the simulations. All the models, both static and dynamic, give a very close evolution for the contact line position. The main difference with experiments is due to the oscillations observed at the end of the drop spreading. Such oscillations are induced by the propagation of capillary wave resulting in some cases of a droplet ejection $[26,23]$. In the simulations, the top of the drop is free to 

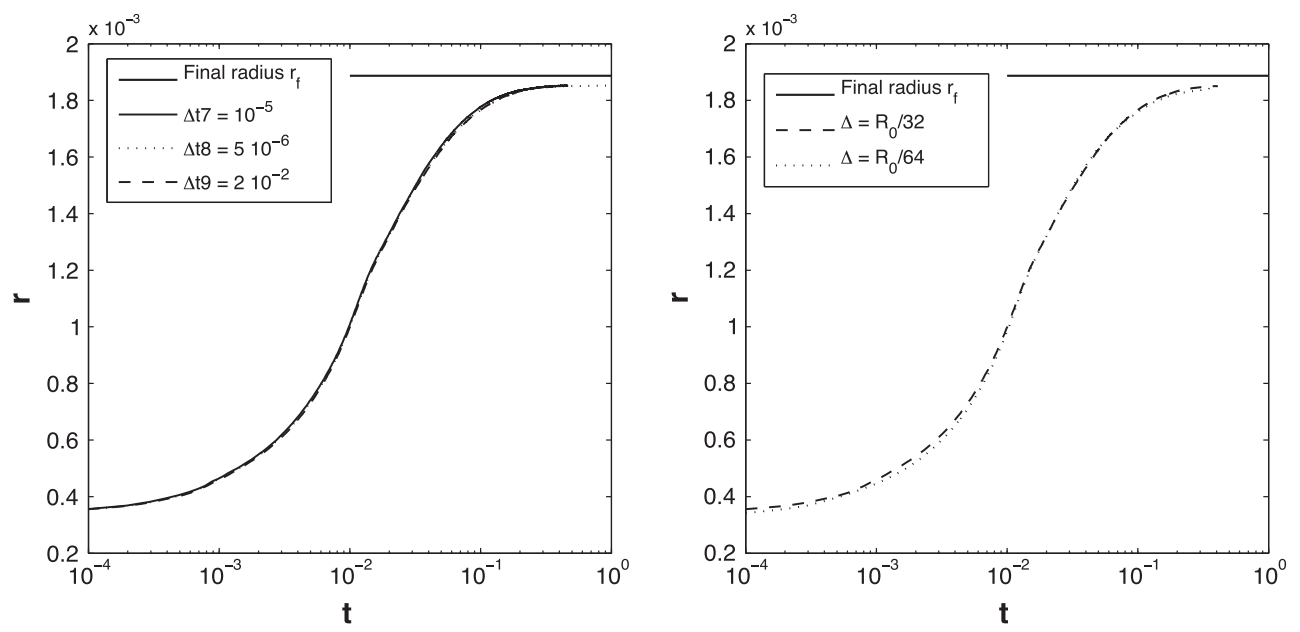

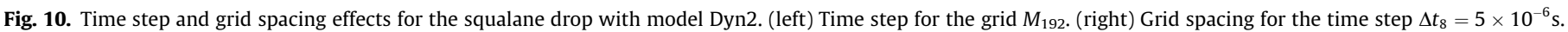
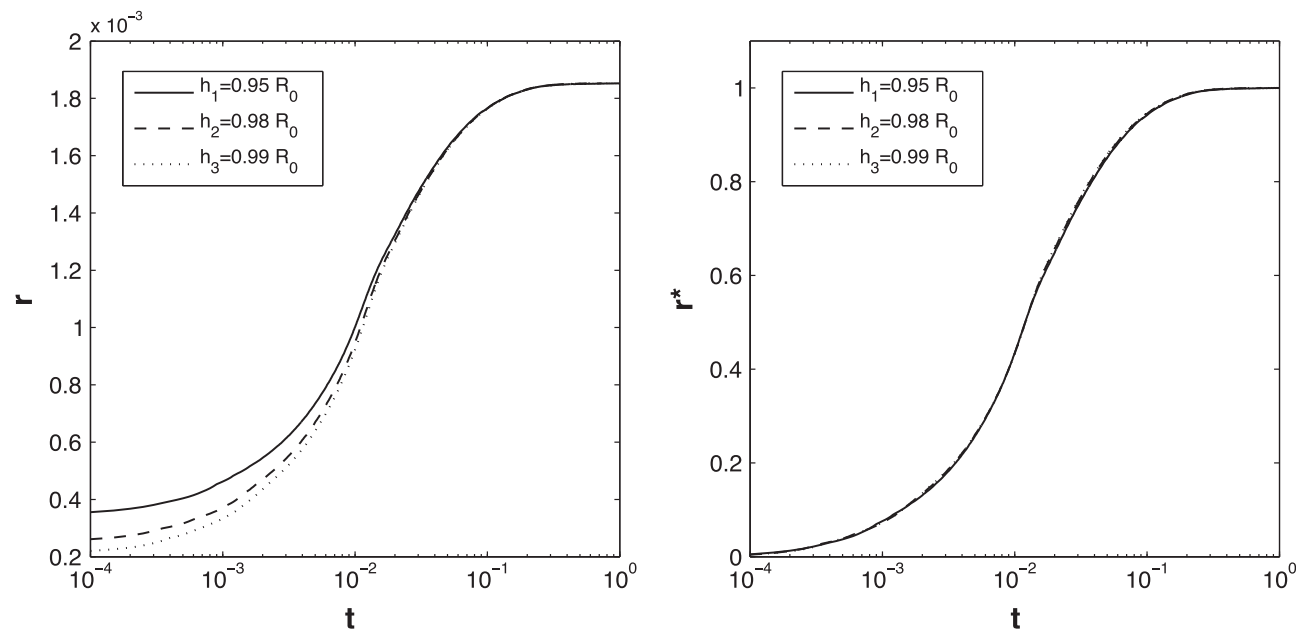

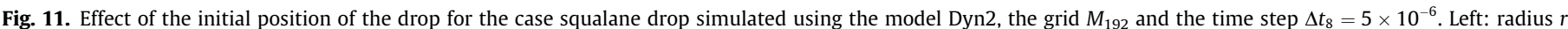
versus time $t$. Right: normalized radius $r^{*}=\left(r-r_{0}\right) /\left(r_{f}-r_{0}\right)$ versus time $t$.

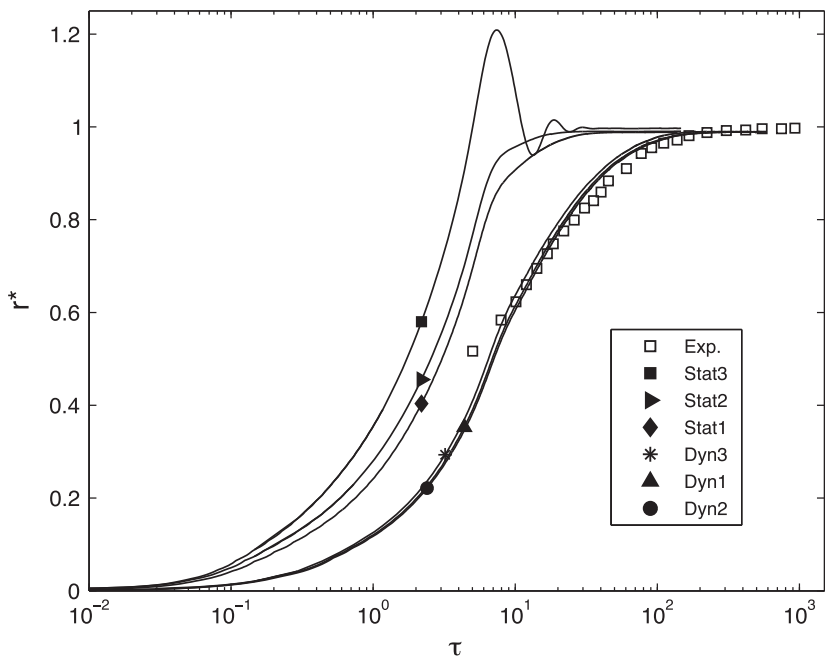

Fig. 12. Comparison with the experiments for the squalane drop. The normalized radius $r *$ is reported versus the normalized time $t / t_{\mu}$. $\square$ experiments from Lavi and Marmur [24].

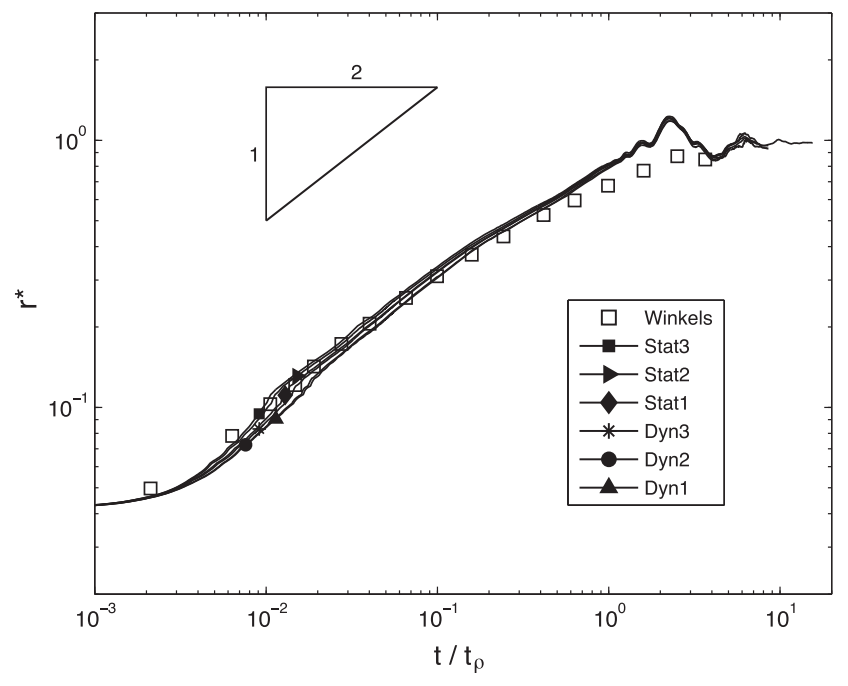

Fig. 13. Contact line radial position $r / R_{0}$ versus the time normalized by the capillary-inertia characteristic time $t_{\rho}=\left(\rho R_{0}^{3} / \sigma\right)^{1 / 2}$. Comparison between the models for $\theta_{S}=115^{\circ}$. $\square$ experiments from [25]. 
move and oscillate due to the capillary waves while in the experiments the drop remains pinned to the needle used for the deposit.

Compared to the viscous spreading, the evolutions obtained using the different models are now very similar. In order to discuss the effect of the drop viscosity on the difference observed between the models, additional simulations have been performed. The viscosity, surface tension and the value of the static contact angle have been independently changed. The dynamic model Dyn2 is compared to the static model Stat3 since it corresponds to the fasted spreading evolution (see Fig. 12).

The difference between the two models is measured by comparing the time $t_{05}$ necessary to reach one half of the final radius $r_{f}$, i.e. $r\left(t_{05}\right)=r_{f} / 2$. The relative difference $\Delta t_{05}=\left(t_{05}^{\text {Dyn2 }}-t_{05}^{\text {Stat3 }}\right) / t_{05}^{\text {Dyn2 }}$ between the times $t_{05}^{\text {Dyn } 2}$ and $t_{05}^{\text {Stat } 3}$ obtained by using the two models Dyn2 and Stat3, respectively, is reported in Fig. 14 as a function of the Ohnesorge number $O h=\mu / \sqrt{\rho \sigma R_{0}}$ for different values of the contact angle. The value of $\Delta t_{05}$ deduced from the simulations shown in Figs. 12 and 13 are also reported. The figure clearly indicates that $\Delta t_{05}$, i.e. the difference between the two models, increases significantly with the Ohnesorge number. For large Ohnesorge numbers, the use of a dynamic model is necessary to capture the correct spreading of a drop while static and dynamic models give similar evolutions for low Ohnesorge numbers.

As an illustration, we reports in Fig. 15 the evolution of a drop spreading for $O h=0.053\left(\mu=0.01\right.$ Pa s, $\sigma=0.072 \mathrm{~N} / \mathrm{m}, \rho=10^{3}$ $\mathrm{kg} / \mathrm{m}^{3}$ and $R_{0}=0.5 \mathrm{~mm}$ ). The static model Stat 3 and the dynamic model Dyn2 are compared. This case corresponding to a fluid ten times more viscous than water has been chosen because the spreading is characterized by two very different evolutions for $\theta_{S}=5^{\circ}$ [23]. The capillary-inertial evolution $r \sim t^{1 / 2}$ is observed at the beginning of the spreading while the end of the spreading is characterized by the famous Tanner law $r \sim t^{1 / 10}$. The simulations have been performed here for a larger contact angle, $\theta_{S}=10^{\circ}$. The two regimes $r \sim t^{1 / 2}$ and $r \sim t^{1 / 10}$ are reproduced when using the dynamic model. For the static model, the capillary-inertial evolution $r \sim t^{1 / 2}$ is observed before the oscillation of the contact line. The Tanner regime is not reproduced. In fact, the Tanner regime is observed when the following two point are combined: (i) the shape of the drop is a very thin spherical cap so that the contact angle $\theta_{W}$, the drop volume $V$ and the contact line radius $r$ are linked by the geometrical relation $\theta_{W} \approx 4 V / \pi r^{3}$; (ii) in the limit of a small dynamic contact angle with $\theta_{S} \approx 0$, the

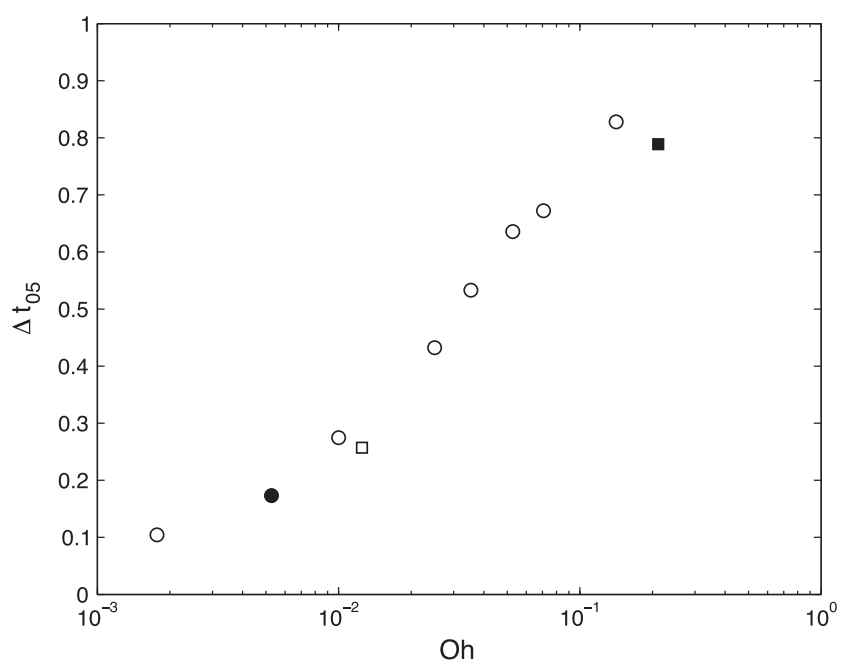

Fig. 14. Evolution of $\Delta t_{05}$ as a function of the Ohnesorge number. $\circ \theta_{S}=115^{\circ}, \square$ $\theta_{S}=65^{\circ}, \boldsymbol{\square}$ and $\bullet$ : values deduced from the simulations shown in Figs. 12 and 13, respectively.

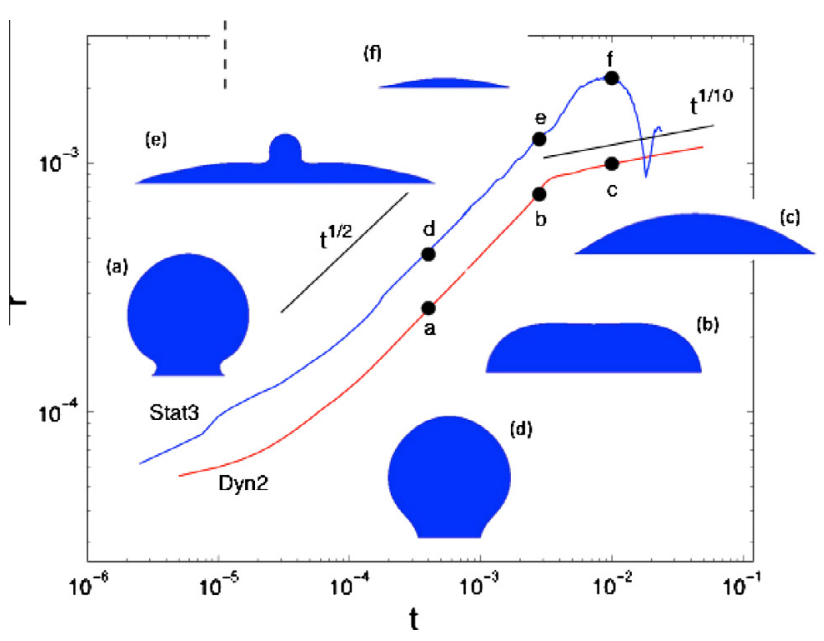

Fig. 15. Effect of the contact angles model on the time evolution of the drop base radius $r$ for $\theta_{S}=10^{\circ}$ and $O h=0.053\left(\mu=0.01 \mathrm{~Pa} \mathrm{~s}, \sigma=0.072 \mathrm{~N} / \mathrm{m}, \rho=10^{3} \mathrm{~kg} / \mathrm{m}^{3}\right.$ and $R_{0}=0.5 \mathrm{~mm}$ ). Drop radial sections are reported for time $t=4 \times 10^{-4} \mathrm{~s}$ (a and d) corresponding to the middle of the $t^{1 / 2}$ evolution, for time $t=2.8 \times 10^{-3} \mathrm{~s}$ (b and e) corresponding to the transition between the $t^{1 / 2}$ and the $t^{1 / 10}$ evolutions, and time $t=10^{-2} \mathrm{~s}$ ( $\mathrm{c}$ and $\mathrm{f}$ ) corresponding to the Tanner law evolution. In (f) the drop has a toroidal shape and the axis of symmetry is shown.

Cox relation (7) can be expressed as $\theta_{W}^{3}=9 \frac{\mu}{\sigma} \frac{d r}{d t} \ln (L / \lambda)$. Combining these two relations and integrating in time, the radius of the contact line is found to follow the Tanner law $r \sim t^{1 / 10}$. The drop shape at different characteristic times of the evolution is reported in Fig. 15. At the beginning of the spreading, the shapes obtained using the two models are very similar. The difference is due to a faster spreading for the static model clearly visible at the drop base. The difference is more significant at the transition between the capillary-inertial regime and the Tanner regime. When using the static model, the drop center dewet resulting in the formation of a toroidal drop that spreads and then retracts. The center is then wet again and finally the drop stabilizes. This explains the large scale oscillation of the contact line. The use of the dynamic model reproduce the Tanner regime characterized by a spherical cap.

\section{Discussion}

One important challenge for the simulation of moving contact lines is the development of predictive numerical models, i.e. models with no adjustable parameter. A full direct numerical simulation would consist in simulating all the scales involved in the problem, i.e. from the slip length at the wall up to the droplet size. For example, let us consider a millimeter-size droplet $R \approx 1 \mathrm{~mm}$ as considered in the experiments used in this study for the validation of the simulations. Nanoscale slip lengths $\lambda$ being usually reported, a full resolution of all the hydrodynamic scales present in this problem requires $N_{2 D} \approx(R / \lambda)^{2} \approx 10^{+12}$ nodes for a 2 D axisymmetric simulation and $N_{3 D} \approx(R / \lambda)^{3} \approx 10^{+18}$ nodes for a full 3D simulation. Such simulations are obviously not accessible with the actual computers. Molecular Dynamics (MD) is an approach that permits to explore the molecular details of wetting. The practical application of MD is limited to very small systems of few tens of nanometers and few nanoseconds [25]. Consequently, it is clear that simulations of contact lines hydrodynamic for millimeter size interfaces can not be performed by solving all the length scales and a cut-off length is imposed by the grid size. However, wetting phenomena are governed by interactions up to the nano-scale. As a consequence, a sub-grid model as described in Dupont and Legendre [10] has to be introduced in order to make possible macroscopic simulations. Based on this approach, the modeling developed in recent works $[23,21,17]$ appears to be the more 
consistent approach for the simulation of moving contact line at the macroscopic scale:

- the wall condition seen by the fluid is a non slip condition,

- the interface shape is connected by a dynamic relation to the characteristics of the contact line at the nanoscale.

Thanks to the hydrodynamic theories [16], the macroscale apparent angle can be directly linked to the static angle and the slip length $\lambda$, making the corresponding expression (see Eq. (11)) very useful for such approach. In this relation, $L$ is the macroscopic cut-off length scale imposed by the grid resolution. The first node being located at $\Delta / 2$ from the wall, it follows that $L=\Delta / 2$. Considering the value of observed slip lengths, we have used in this paper a fixed value $\lambda=10^{-9} \mathrm{~m}$ and the simulations seem to correctly reproduce the experiments for both small and large Ohnesorge numbers. The value chosen for $\lambda$ can obviously be adapted to any surface of known slip length. However, as commented in [21] where numerical simulations of 3D sliding drops are compared with experiments, it is not clear if such modeling is able to correctly describe the dissipation induced by moving contact lines. Additional investigations are still necessary to clarify this point.

\section{Conclusion}

Different models (static and dynamic) for the description of moving contact lines have been introduced in a CSF-VoF method. The results obtained for spreading drop are found to be very sensible to the choice of the model. The difference can be significant between dynamic and static models as well as between no-slip and numerical slip conditions. A time convergence can be reached for all the models considered. We have observed that the time step required for the time convergence depends on the grid spacing. It is also clear that a grid convergence can only be perfectly obtained when the imposed numerical slip is solved by an adapted grid. Interestingly, the use of the Cox-Voinov dynamic model improves the grid convergence compared to a static description of the contact angle. A perfect grid convergence can not be reached with such modeling since the viscous stress at the contact line is still diverging with the grid refinement. The model proposed by Afkhami et al. [14] does not provide the grid convergence expected considering the simulations shown by the authors. We have also tested this model for the 2D situation since it has been adjusted for 2D spreading but the grid convergence is not improved. Since the model is adjusted using simulations, it may be dependent on the method used to solve the system of Eqs. (3)-(6). This can be a possible explanation since our study reveals that the initial acceleration of the contact line is strongly grid dependent during the first times of the simulation. We have shown that it results from the finite volume integration of the CSF formulation. The static and dynamic models have been compared to experiment for the spreading of both water and squalane drops. The difference between static and dynamic models is increased when increasing the Ohnesorge number. Static models are able to reproduce the inertial-capillary spreading $r \sim t^{1 / 2}$ but are not able to capture the Tanner evolution $r \sim t^{1 / 10}$ because this regime directly results from the evolution of the dynamic angle with the contact line velocity.

\section{Acknowledgement}

This research project has been financed by a Marie Curie Early Stage Research Training Fellowship of the European Community sixth Framework Program under the Contract Number MEST-CT-2005-020426.

\section{References}

[1] Schleizer A, Bonnecaze R. Displacement of a two-dimensional immiscible droplet adhering to a wall in shear and pressure driven flow. J Fluid Mech 1999;383:29-54.

[2] Dimitrakopoulos P, Higdon J. On the gravitational displacement of three dimensional fluid droplets from inclined solid surfaces. J Fluid Mech 1999;295:181-209.

[3] Glasner K. A boundary integral formulation of quasi-steady fluid wetting. J Comput Phys 2005;207:529-41.

[4] Finlow DE, Kota PR, Bose A. Investigation of wetting hydrodynamics using numerical simulations. Phys Fluids 1996;8:302.

[5] Shen C, Ruth D. Experimental and numerical investigations of the interface profile close to a moving contact line. Phys Fluids 1998;10:789.

[6] Spelt PDM. A level-set approach for simulations of flows with multiple moving contact lines with hysteresis. J Comput Phys 2005;207: 389-404.

[7] Liu H, Krishnan S, Marella S, Udaykumar H. Sharp interface cartesian grid method II: A technique for simulating droplets interactions with surfaces of arbitrary shape. J Comput Phys 2005;210:32-54.

[8] Renardy M, Renardy Y, Li J. Numerical simulation of moving contact line problems using a volume-of-fluid method. J Comput Phys 2001;94.

[9] Afkhami S, Bussmann M. Heigh functions for applying contact angles to 2D VOF simulations. Int J Numer Metods Fluids 2008;57:453-72.

[10] Dupont J, Legendre D. Numerical simulations of static and sliding drop with contact angle hysteresis. J Comput Phys 2010;229:2453-78.

[11] Khenner M. Computation of the material indicator function near the contact line (in Tryggvason's method). J Comput Phys 2004;200:1-7.

[12] Yamamoto Y, Uemura T. Dynamic contact angle of a spreading drop represented by front-tracking simulation. In: 7th International conference on multiphase flow 2010.

[13] Yokoi K, Vadillo D, Hinch J, Hutchings I. Numerical studies of the influence of the dynamic contact angle on a droplet impacting on a dry surface. Phys Fluids $2009 ; 21$.

[14] Afkhami S, Zaleski S, Bussmann M. A mesh-dependent model for applying dynamic contact angles to VOF simulations. J Comput Phys 2009;228: 5370-89.

[15] Bonn D, Eggers J, Indekeu J, Meunier J, Rolley E. Wetting and spreading. Rev Modern Phys 2009;81.

[16] Cox R. The dynamics of the spreading of liquids on a solid surfaces. Part 1: Viscous flow. J Fluid Mech 1986;168.

[17] Sui Y, Spelt P. An efficient computational model for macroscale simulations of moving contact lines. J Comput Phys 2013;242:37-52.

[18] Bonometti T, Magnaudet J. An interface capturing method for incompressible two-phase flows. Validation and application to bubble dynamics. Int J Multiphase Flow 2007;33:109-33.

[19] Zalesak S. Fully multidimensional flux-corrected transport algorithms for fluids. J Comput Phys 1979;31:335-62.

[20] Brackbill J, Kothe D, Zemach C. A continuum method for modeling surface tension. J Comput Phys 1992;100:335-54.

[21] Maglio M, Legendre D. Numerical simulation of sliding drops on an inclined solid surface. In: Sigalotti Leonardo, Klapp Jaime, Sira Eloy, editors. International Publishing AG; 2013. p. 1-14.

[22] Lauga E, Brenner M, Stone H. Microfluidics: the no-slip boundary condition. In: Handbook of experimental fluid dynamics. New York: Springer; 2007.

[23] Legendre D, Maglio M. Numerical simulation of spreading drops. Colloids Surf A: Physicochem Eng Aspects 2013;432:29-37.

[24] Lavi B, Marmur A. The exponential power law: partial wetting kinetics and dynamic contact angles. Colloids Surf 2004;250.

[25] Winkels K, Weijs J, Eddi A, Snoeijer J. Initial spreading of low-viscosity drops on partially wetting surfaces. Phys Rev E 2012;85:055301 (R).

[26] Ding H, Li E, Zhang F, Sui Y, Spelt P, Thoroddsen S. Propagation of capillary waves and ejection of small droplets in rapid droplet spreading. J Fluid Dyn 2012;697:92-114. 